\title{
The synergetic effect of Imipenem- clarithromycin combination in the Mycobacteroides abscessus complex
}

Satomi Takei ${ }^{1}$, Hiroaki Ihara ${ }^{2,3^{*}+}$ D, Shinsaku Togo ${ }^{2,4^{*}+}$, Ayako Nakamura ${ }^{5}$, Yuichi Fujimoto ${ }^{2}$, Junko Watanabe ${ }^{2}$, Kana Kurokawa ${ }^{2}$, Kohei Shibayama ${ }^{2}$, Issei Sumiyoshi ${ }^{2}$, Yusuke Ochi ${ }^{2}$, Moe Iwai ${ }^{2,4}$, Takahiro Okabe ${ }^{6}$, Masayoshi Chonan', Shigeki Misawa', Akimichi Ohsaka and Kazuhisa Takahashi ${ }^{2}$

\begin{abstract}
Background: Nontuberculous mycobacteria (NTM) are ubiquitous organisms and the incidence of NTM infections has been increasing in recent years. Mycobacteroides abscessus (M. abscessus) is one of the most antimicrobialresistant NTM; however, no reliable antibiotic regimen can be officially advocated. We evaluated the efficacy of clarithromycin in combination with various antimicrobial agents against the M. abscessus complex.

Results: Twenty-nine clinical strains of $M$. abscessus were isolated from various clinical samples. Of the isolates, 10 (34.5\%) were of $M$. abscessus subsp. abscessus, 18 (62.1\%) of $M$. abscessus subsp. massiliense, and 1 (3.4\%) of $M$. abscessus subsp. bolletii. MICs of three antimicrobial agents (amikacin, imipenem, and moxifloxacin) were measured with or without clarithromycin. The imipenem-clarithromycin combination significantly reduced MICs compared to clarithromycin and imipenem monotherapies, including against resistant strains. The association between susceptibility of the M. abscessus complex and each combination of agents was significant $(p=0.001)$. Adjusted residuals indicated that the imipenem-clarithromycin combination had the synergistic effect (adjusted residual = 3.1) and suppressed the antagonistic effect (adjusted residual $=-3.1$ ). In subspecies of M. abscessus complex, the association with susceptibility of $M$. abscessus subsp. massiliense was similarly statistically significant ( $p=0.036$ : adjusted residuals of synergistic and antagonistic effect respectively: 2.6 and -2.6$)$. The association with susceptibility of M. abscessus subsp. abscessus also showed a similar trend but did not reach statistical significance.
\end{abstract}

Conclusion: Our data suggest that the imipenem-clarithromycin combination could be the recommended therapeutic choice for the treatment of $M$. abscessus complex owing to its ability to restore antimicrobial susceptibility.

Keywords: Clarithromycin, Fractional inhibitory concentration index, Imipenem, Mycobacteroides abscessus

\footnotetext{
* Correspondence: h-ihara@juntendo.ac.jp; shinsaku@juntendo.ac.jp

${ }^{+}$Hiroaki Ihara and Shinsaku Togo contributed equally to this work.

${ }^{2}$ Department of Respiratory Medicine, Juntendo University, Faculty of

Medicine \& Graduate School of Medicine, 2-1-1 Hongo, Bunkyo-Ku, Tokyo

113-8421, Japan

Full list of author information is available at the end of the article
}

(c) The Author(s). 2020 Open Access This article is licensed under a Creative Commons Attribution 4.0 International License, which permits use, sharing, adaptation, distribution and reproduction in any medium or format, as long as you give appropriate credit to the original author(s) and the source, provide a link to the Creative Commons licence, and indicate if changes were made. The images or other third party material in this article are included in the article's Creative Commons licence, unless indicated otherwise in a credit line to the material. If material is not included in the article's Creative Commons licence and your intended use is not permitted by statutory regulation or exceeds the permitted use, you will need to obtain permission directly from the copyright holder. To view a copy of this licence, visit http://creativecommons.org/licenses/by/4.0/ The Creative Commons Public Domain Dedication waiver (http://creativecommons.org/publicdomain/zero/1.0/) applies to the data made available in this article, unless otherwise stated in a credit line to the data. 


\section{Background}

NTM are ubiquitous organisms that cause diverse types of infectious diseases in humans, including in lungs, the lymphatic system, skin, soft tissue, bone disease, and are disseminated. The morbidity of NTM has been increasing worldwide [1, 2]; the 2014 nationwide survey of NTM in Japan revealed that the incidence of pulmonary NTM (14.7 cases/100,000 person/year) has overtaken that of tuberculosis (12.9 cases/100,000 person/year) [3]. Above all, the Mycobacterium avium complex (88.8\%) were the most frequently isolated organisms, followed by Mycobacterium kansasii (4.3\%) and the Mycobacteroides abscessus complex (3.3\%). Notably, the incidence of $M$. abscessus-infected pulmonary disease has dramatically increased in Japan, from 0.1 cases/100,000 person/year in 2001 to 0.5 cases/100,000 person/year in 2014. M. abscessus is one of the treatment-refractory NTM, characterized by rapid growth and multidrug resistance. $M$. abscessus, which is frequently isolated from respiratory tracts of patients with cystic fibrosis (CF), has been the leading cause of rapid growing mycobacteria in CF since the 2000s [4, 5]. The critical features of $M$. abscessus involve resistance to most antibiotics in clinical use, including first line antitubercular drugs [6, 7]. The 2007 American Thoracic Society/Infectious Diseases Society of America (ATS/IDSA) statement recommended multidrug therapy, including a macrolide and one or more parenteral agents (e.g., amikacin, cefoxitin, or imipenem) [8]; however, recommendations for the treatment of $M$. abscessus are known to be of limited efficacy [9]. Recently, three subspecies of $M$. abscessus have been defined: $M$. abscessus subsp. abscessus, M. abscessus subsp. bolletii, and M. abscessus subsp. massiliense. $M$. abscessus subsp. massiliense specifically lacks the erm (41) gene associated with macrolide resistance, and thus, the macrolide susceptibility among $M$. abscessus subsp. massiliense and M. abscessus subsp. abscessus and bolletii is different $[10,11]$. For this reason, some experts recommend non-macrolide combinations for treatment for macrolide-resistant $M$. abscessus subspecies, based on identified in vitro susceptibilities [12]. Here, we propose new insights into the synergistic effects on $M$. abscessus susceptibility achieved in vitro by clarithromycin in combination with other antimicrobials.

\section{Results}

\section{Clinical features of three subspecies of M. abscessus complex}

Twenty-nine clinical strains of $M$. abscessus were isolated from various clinical samples at the Juntendo university hospital from 2011 to 2019. The characteristics of patients from which $M$. abscessus complex were isolated are shown in Table 1. Twenty-two of 29 (75.9\%) patients were diagnosed with $M$. abscessus complex from
Table 1 The characteristics of patients from which M. abscessus complex were isolated

\begin{tabular}{ll}
\hline & $\mathbf{N}=\mathbf{2 9}$ \\
\hline Sex (Male/Female) & $12 / 17$ \\
Median age (range) & $65(38-83)$ \\
Smoking history, N (\%) & $9(31.0)$ \\
M.abscessus complex subtype, N (\%) & \\
M.abscessus subsp. abscessus & $10(34.5)$ \\
M.abscessus subsp. masiliense & $18(62.1)$ \\
M.abscessus subsp. bolletii & $1(3.4)$ \\
M.abscessus complex detected from, N (\%) & \\
Sputum or bronchial lavage & $22(75.9)$ \\
Others & $7(24.1)$ \\
erm (41) gene status, N (\%) & \\
deletion & $18(17.2)$ \\
T28 & $9(31.0)$ \\
C28 & $2(6.9)$ \\
Pretreatment of antibiotics within 3 months, N (\%) & \\
Macrolides & $5(17.2)$ \\
Fluoroquinolones & $5(17.2)$ \\
Tetracyclines & $2(6.9)$ \\
Others & $12(41.4)$ \\
Bronorbidity, N (\%) & \\
Diabetes mellitus & \\
Mmmunodeficiency (non HIV) & $10(34.5)$ \\
Concomitant medications, N (\%) & $4(13.8)$ \\
\hline Abbricosteroids & $2(13.8)$ \\
\hline
\end{tabular}

Abbreviations: HIV human immunodeficiency virus

the culturing of sputum or bronchial lavage. Of the isolates, 10 (34.5\%) were of M. abscessus subsp. abscessus, 18 (62.1\%) of $M$. abscessus subsp. massiliense, and 1 (3.4\%) of M. abscessus subsp. bolletii as determined by multi-locus sequence analysis. The treatment history indicated that 24 of $29(82.8 \%)$ patients had received antibiotics in the last 3 months, including macrolides, and 10 of 29 (34.5\%) patients had received immunosuppressive treatment including corticosteroids before the culture. erm (41) sequevars affected clarithromycin susceptibility in $M$. abscessus, namely, erm (41) with a large deletion and C28 sequevar related to susceptibility, and with T28 sequevar related to resistance. All 18 strains of $M$. abscessus subsp. massiliense possessed erm (41) with a large deletion and only 2 strains of $M$. abscessus subsp. abscessus had erm (41) with C28 sequevar. 


\section{Susceptibility to antimicrobial agents in combination with clarithromycin}

MICs on day 3, 7, and 14 were recorded (Fig. 1, S1, and S2). Bacterial growth on day 3 was insufficient. MIC on day 14 was equivalent as compared to MICs on day 7; however, over-growth was observed after 14-day incubation. Thus, MICs on day 7 were used in following experiments. The susceptibility to a combination of clarithromycin and antimicrobial agents was compared to that of the antimicrobial agents alone, categorized into each subspecies of M. abscessus complex (Fig. 1). The MICs of three antimicrobial agents (amikacin, imipenem, and moxifloxacin) were measured with or without clarithromycin. Notably, the use of imipenemclarithromycin combination mostly reduced the MIC of imipenem. The combination also reduced the MIC of clarithromycin, even in clarithromycin-resistant subspecies of $M$. abscessus complex. The effect of reducing clarithromycin MICs by the imipenem-clarithromycin combination was stronger than that of the amikacinand moxifloxacin-clarithromycin combination. In 1 strain of $M$. abscessus subsp. abscessus and 3 strains of M. abscessus subsp. massiliense, susceptibility was not restored by the combined use of clarithromycin and imipenem, and only 1 strain of $M$. abscessus subsp. bolletii did not respond to any combination. MICs of clarithromycin alone in $M$. abscesssus complex with a large deletion and C28 sequevar in erm (41) were significantly lower than that of T28 sequevar $(p=0.0075)$. In subspecies of $M$. abscessus complex, MICs of imipenem and clarithromycin in combination were significantly less than that of either clarithromycin or imipenem alone in both $M$. abscessus subsp. massiliense and abscessus ( $p<$ 0.001 for both subspecies) (Table S1).

We next determined the synergistic effect of the imipenem-clarithromycin combination as compared to amikacin- or moxifloxacin-clarithromycin combinations, using the fractional inhibitory concentration (FIC) index as described in previous paper [13] (Fig. 2). Susceptibility was divided into two classes, synergy and additive as a synergistic effect and indifference and antagonism as an antagonistic effect. Table 2 showed the number of

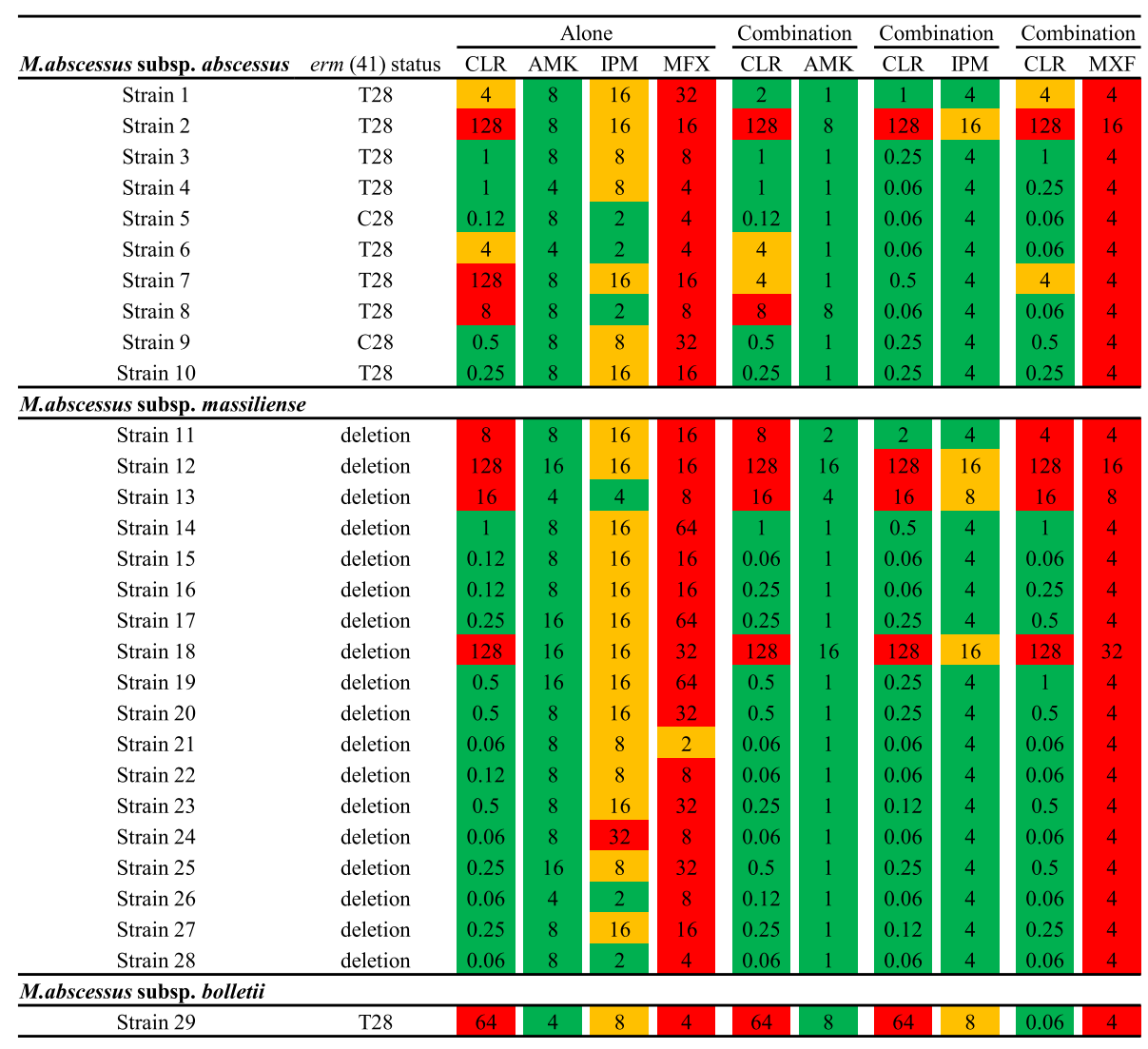

\begin{tabular}{|l|l|l|}
\hline Susceptible & Intermediate & Resistant \\
\hline
\end{tabular}

Fig. 1 MIC distributions for amikacin, imipenem, and moxifloxacin combined with clarithromycin, categorized into three subspecies of $M$. abscessus complex on day 7. Green color indicates susceptibility, yellow color indicates intermediate, and red color indicates resistance to $M$. abscessus. Abbreviations: CLR, clarithromycin; AMK, amikacin; IPM, imipenem; MXF, moxifloxacin 


\begin{tabular}{|c|c|c|c|c|}
\hline M.abscessus subsp. abscessus & erm (41) & CLR/AMK & CLR/IPM & $\mathrm{CLR} / \mathrm{MXF}$ \\
\hline Strain 1 & $\mathrm{~T} 28$ & 0.625 & 0.5 & 1.125 \\
\hline Strain 2 & $\mathrm{~T} 28$ & 2 & 2 & 2 \\
\hline Strain 3 & $\mathrm{~T} 28$ & 1.125 & 0.75 & 1.5 \\
\hline Strain 4 & $\mathrm{~T} 28$ & 1.25 & 0.56 & 1.25 \\
\hline Strain 5 & $\mathrm{C} 28$ & 1.125 & 2.5 & 1.5 \\
\hline Strain 6 & $\mathrm{~T} 28$ & 1.25 & 2.015 & 1.015 \\
\hline Strain 7 & $\mathrm{~T} 28$ & 0.156 & 0.254 & 0.281 \\
\hline Strain 8 & $\mathrm{~T} 28$ & 2 & 2.008 & 0.508 \\
\hline Strain 9 & $\mathrm{C} 28$ & 1.125 & 1 & 1.125 \\
\hline Strain 10 & $\mathrm{~T} 28$ & 1.125 & 1.25 & 1.25 \\
\hline \multicolumn{5}{|l|}{ M.abscessus subsp. massiliense } \\
\hline Strain 11 & deletion & 1.25 & 0.5 & 0.75 \\
\hline Strain 12 & deletion & 2 & 2 & 2 \\
\hline Strain 13 & deletion & 2 & 3 & 2 \\
\hline Strain 14 & deletion & 1.125 & 0.75 & 1.063 \\
\hline Strain 15 & deletion & 0.625 & 0.75 & 0.75 \\
\hline Strain 16 & deletion & 2.208 & 0.75 & 2.333 \\
\hline Strain 17 & deletion & 1.063 & 1.25 & 2.063 \\
\hline Strain 18 & deletion & 2 & 2 & 2 \\
\hline Strain 19 & deletion & 1.063 & 0.75 & 2.063 \\
\hline Strain 20 & deletion & 1.125 & 0.75 & 1.125 \\
\hline Strain 21 & deletion & 1.125 & 1.5 & 3 \\
\hline Strain 22 & deletion & 0.625 & 1 & 1 \\
\hline Strain 23 & deletion & 0.625 & 0.49 & 1.125 \\
\hline Strain 24 & deletion & 1.125 & 1.125 & 1.5 \\
\hline Strain 25 & deletion & 2.063 & 1.5 & 2.125 \\
\hline Strain 26 & deletion & 2.25 & 3 & 1.5 \\
\hline Strain 27 & deletion & 1.125 & 0.73 & 1.25 \\
\hline Strain 28 & deletion & 1.125 & 3 & 2 \\
\hline \multicolumn{5}{|l|}{ M.abscessus subsp. bolletii } \\
\hline Strain 29 & $\mathrm{~T} 28$ & 3 & 2 & 1.001 \\
\hline & & \multicolumn{2}{|c|}{ FIC index $\leqq 0.5$} & Synergy \\
\hline & & \multicolumn{2}{|c|}{$0.5<$ FIC index $\leqq 1$} & Additive \\
\hline & & \multicolumn{2}{|c|}{$1<$ FIC inde $\leqq 2$} & ndifference \\
\hline & & \multicolumn{2}{|c|}{$2<$ FIC index } & Antagonism \\
\hline
\end{tabular}

Fig. 2 FIC index of amikacin, imipenem, and moxifloxacin combined with clarithromycin, categorized into three subspecies of M. abscessus. Light green color indicates synergy, green color indicates additive, yellow color indicates indifference, and red color indicates antagonism in each combination. Abbreviations: CLR, clarithromycin; AMK, amikacin; IPM, imipenem; MXF, moxifloxacin; FIC index, fractional inhibitory concentration index

isolates showing synergistic and antagonistic effects with each combination. In $M$. abscessus complex, 14 strains (48.3\%) revealed synergistic effects for imipenem-clarithromycin combination, in contrast, only 5 strains $(17.2 \%)$ for moxifloxacin- or amikacinclarithromycin combination. In $M$. abscessus subsp. massiliense, 9 strains (50.0\%) revealed synergistic effects for imipenem-clarithromycin combination, only 3 strains (16.7\%) for moxifloxacin- or amikacinclarithromycin combination. In $M$. abscessus subsp. abscessus, 5 strains (50.0\%) revealed synergistic effects for imipenem-clarithromycin combination, only 2 strains (20.0\%) for moxifloxacin- or amikacinclarithromycin combination. 
Table 2 The number of synergistic and antagonistic combination with clarithromycin and each antimicrobia

\begin{tabular}{|c|c|c|c|c|c|}
\hline \multirow[b]{2}{*}{ Species } & \multirow[b]{2}{*}{ Categories of FIC index } & CLR/AMK & CLR/IPM & \multirow[t]{2}{*}{ CLR/MXF } & \multirow[b]{2}{*}{$p$ value } \\
\hline & & \multicolumn{2}{|c|}{$\mathrm{N}$ (\%, Adjusted residual) } & & \\
\hline M.abscessus complex & Synergy + Additive & $5(17.2,-1.5)$ & $14\left(48.3,3.1^{* *}\right)$ & $5(17.2,-1.5)$ & $0.001^{* *}$ \\
\hline$N=29+$ & Indifference + Antagonism & $24(82.8,1.5)$ & $15\left(51.7,-3.1^{* *}\right)$ & $24(82.8,1.5)$ & \\
\hline M. abscessus subsp. massiliense & Synergy + Additive & $3(16.7,-1.3)$ & $9\left(50.0,2.6^{* *}\right)$ & $3(16.7,-1.3)$ & $0.036^{*}$ \\
\hline$N=18$ & Indifference + Antagonism & $15(83.3,1.3)$ & $9\left(50.0,-2.6^{* *}\right)$ & $15(83.3,1.3)$ & \\
\hline M. abscessus subsp. abscessus & Synergy + Additive & $2(20.0,-0.8)$ & $5(50.0,1.7)$ & $2(20.0,-0.8)$ & 0.24 \\
\hline$N=10$ & Indifference + Antagonism & $8(80.0,0.8)$ & $5(50.0,-1.7)$ & $8(80.0,0.8)$ & \\
\hline
\end{tabular}

† including M. abscessus subsp. boletii $(\mathrm{n}=1)$

${ }^{*} p$ value $<0.05,{ }^{* *} p$ value $<0.01$

$*$ adjusted residuals $>|1.96|,{ }^{* *}$ adjusted residuals $>|2.58|$

Abbreviations: FIC index fractional inhibitory concentration index, CLR clarithromycin, AMK amikacin, IPM imipenem, MXF moxifloxacin

Association of clinical features with susceptibilities to the imipenem-clarithromycin combination

We investigated whether susceptibility to the imipenemclarithromycin combination might associate with clinical status. The isolates from patients with immunosuppression and/or administered immunosuppressive drugs and/or corticosteroids revealed synergistic effects rather than antagonistic effects $(p=0.040)$ (Table 3$)$. The other clinical parameters such as age, sex, smoking history, bronchiectasis lesion, a treatment history of antibiotics, and erm (41) gene status did not influence the effects of imipenem-clarithromycin combination.

\section{Discussion}

We demonstrated here that the MICs of clarithromycin and imipenem were significantly reduced by the

Table 3 The number of synergistic and antagonistic combination with clarithromycin and imipenem in each clinical status

\begin{tabular}{|c|c|c|c|}
\hline & \multicolumn{2}{|l|}{ FIC index } & \multirow[b]{2}{*}{$p$ value } \\
\hline & $\begin{array}{l}\text { Synergy + Additive } \\
N=14(\%)\end{array}$ & $\begin{array}{l}\text { Indifference + Antagonism } \\
N=15(\%)\end{array}$ & \\
\hline \multicolumn{4}{|l|}{ Age } \\
\hline$<65$ years & $7(24.1)$ & $7(24.1)$ & \multirow[t]{2}{*}{0.86} \\
\hline$\geq 65$ years & $7(24.1)$ & $8(27.6)$ & \\
\hline \multicolumn{4}{|l|}{ Sex } \\
\hline Male & $6(20.7)$ & $6(20.7)$ & \multirow[t]{2}{*}{0.88} \\
\hline Female & $8(27.6)$ & $9(31.0)$ & \\
\hline \multicolumn{4}{|c|}{ Smoking history } \\
\hline Yes & $3(10.3)$ & $6(20.7)$ & \multirow[t]{2}{*}{0.43} \\
\hline No & $11(37.9)$ & $9(31.0)$ & \\
\hline \multicolumn{4}{|c|}{ With bronchiectasis } \\
\hline Yes & $4(13.8)$ & $6(20.7)$ & \multirow[t]{2}{*}{0.70} \\
\hline No & $10(34.5)$ & $9(31.0)$ & \\
\hline \multicolumn{4}{|c|}{ With immunosuppression } \\
\hline Yes & $10(34.5)$ & $5(17.2)$ & \multirow[t]{2}{*}{$0.040^{*}$} \\
\hline No & $4(13.8)$ & $10(34.5)$ & \\
\hline \multicolumn{4}{|c|}{ Pretreatment of antibiotics } \\
\hline Yes & $6(20.7)$ & $8(27.6)$ & \multirow[t]{2}{*}{0.57} \\
\hline No & $8(27.6)$ & $7(24.1)$ & \\
\hline \multicolumn{4}{|c|}{ erm (41) gene status } \\
\hline $\mathrm{del}+\mathrm{C} 28$ & $10(34.5)$ & $10(34.5)$ & \multirow[t]{2}{*}{0.78} \\
\hline T28 & $4(13.8)$ & $5(17.2)$ & \\
\hline
\end{tabular}

Antibiotics including clarithromycin $(n=3)$

$p$ value $<0.05, * * 0$ value $<0.01$

Abbreviations: FIC index fractional inhibitory concentration index, del deletion 
administration of an imipenem-clarithromycin combination. We propose a new therapeutic benefit by which the imipenem-clarithromycin combination could reduce the MICs of M. abscessus isolates showing resistance to clarithromycin and/or imipenem. The isolates included M. abscessus subsp. abscessus, well known among the three subspecies to show high resistance rate to macrolides $[10,11]$. Furthermore, this combination may be suitable for treatment of $M$. abscessus complex in patients with immunosuppression.

There were several problems involved in the current recommended treatment for $M$. abscessus, due to the lack of clinical outcomes, and uncertain interactions present in multidrug combination therapy; thus, there is still limited reliable evidence to promote a global standard treatment regimen for the three subspecies of $M$. abscessus complex. Previous in vitro studies have demonstrated that treatment with the standard regimen therapy (combinations of clarithromycin, amikacin, and cefoxitin) failed to effectively inhibit the growth of $M$. abscessus due to acquired drug resistance [14]. In vivo, the triple-drug regimen was equally or less effective against $M$. abscessus than cefoxitin alone [15]. A systematic review revealed different outcomes of macrolidecontaining combination regimens against $M$. abscessus subsp. abscessus and massiliense. Macrolide-containing combination regimens for $M$. abscessus subsp. abscessus induced lower rates of negative conversion of sputum culture and higher recurrence rates than that of $M$. abscessus subsp. massiliense [16]. For these reasons, the appropriate drug therapy against $M$. abscessus remains uncertain. $M$. abscessus complex spontaneously produce broad-spectrum $\beta$-lactamases, resulting in reduced susceptibility to $\beta$-lactams, including imipenem. The combination therapy of imipenem with rifabutin or amikacin was more effective than the monotherapy of imipenem against $M$. abscessus complex $[17,18]$. Miyasaka et al. verified the best combined antibiotics with imipenem and described that the imipenem-clarithromycin combination had a high rate of synergistic and additive effects, and revealed a decrease in the MIC values inhibiting $50 \%$ or $90 \%$ of M. abscessus complex [19]. Interestingly, we checked the effect of clarithromycin synergy with each antibiotic, resulting in the same combination therapy. Further, our data revealed the details of Miyasaka's findings to evaluate the effect of imipenem-clarithromycin combination in each subspecies or patient character. Although the exact mechanism for the synergistic effect of clarithromycin combinations was unknown. Therefore, imipenem may be useful in combination with clarithromycin for the treatment of $M$. abscessus complex. Limitations of the present study include the lack of clinical outcomes measured in patients with $M$. abscessus complex treated with imipenem- clarithromycin combination therapy. The number of clinical isolates used in the study was still insufficient when we separately analyzed the susceptibility of $M$. abscessus subspecies. Therefore, we could not demonstrate the synergistic effects of imipenem-clarithromycin combination in $M$. abscessus subsp. abscessus. Further experiments were required to confirm the efficacy of the combination regimen.

\section{Conclusion}

In our in vitro study, we demonstrated the synergistic effect of the imipenem-clarithromycin combination in restoring $M$. abscessus complex antimicrobial susceptibility. Further, this synergistic effect may occur not only in $M$. abscessus subsp. massiliense, but also in $M$. abscessus subsp. abscessus. Thus, our present results suggest that the imipenem-clarithromycin combination could be an effective treatment regimen against both $M$. abscessus subsp. massiliense and M. abscessus subsp. abscessus.

\section{Methods \\ Determination of $M$. abscessus complex}

All material samples suspected of mycobacterial contamination in the Juntendo university hospital were cultured in mycobacteria growth indicator tube (MGIT; Becton Dickinson, USA) broth and incubated at $37^{\circ} \mathrm{C}$ in the BACTEC MGIT 960 (Becton Dickinson, USA) instrument with ambient air. MGIT positive tubes were classified as M. abscessus based on the results of DNA-DNA hybridization (DDH) analysis (DDH Mycobacterium "Kyokuto" kit; Kyokuto Pharmaceutical Industrial, Japan) or matrix-assisted laser-desorption/ionization time-offlight mass spectrometry (MALDI-TOF MS). Detected species were reconfirmed as three subspecies of $M$. abscessus complex by sequencing the $16 S$ rRNA, rpoB, hsp65, and erm genes [20, 21]. All strains of $M$. abscessus were cultured on BD trypticase soy agar II with 5\% sheep blood (Blood agar; Nippon Becton-Dickinson and Company, Japan) at $35^{\circ} \mathrm{C}$ for approximately 4 to 6 days in an aerobic atmosphere. The study protocol was approved by the Ethics Committee of Juntendo University School of Medicine (no. 18-010 and 19-038).

\section{MALDI-TOF MS analysis}

MALDI-TOF MS analysis was performed based on previously described methods [22]. Colonies of M. abscessus complex on blood agar were scratched with a needle, and particles on the needle surface were diluted in $50 \mu \mathrm{L}$ $80 \%$ trifluoroacetic acid. After incubation for $15 \mathrm{~min}$ at room temperature, the solution was added to $150 \mu \mathrm{L}$ distilled water and $200 \mu \mathrm{L} 100 \%$ acetonitrile, followed by a centrifugation step $(16,200 \times \mathrm{g}, 2 \mathrm{~min})$. One microliter of the cleared supernatant containing the bacterial extract was transferred onto a MALDI target plate (Bruker 
Table 4 Forward and backward primers used for PCR

\begin{tabular}{ll}
\hline Target & Sequence \\
\hline 16S rRNA & Forward, 5'-AGA GTT TGA TCM TGG CTC AG-3' \\
& Reverse, 5'-TAC GGT TAC CTT GTT ACG AC-3' \\
rpoB & Forward, 5'-GAG GGT CAG ACC ACG ATG AC -3' \\
& Reverse, 5'-AGC CGA TCA GAC CGA TGT T-3' \\
hsp65 & Forward, 5'ACC AAC GAT GGT GTG TCC AT - 3' \\
& Reverse, 5' CTT GTC GAA CCG CAT ACC CT-3' \\
erm & Forward, 5'-GAC CGG GCC TTC GTG AT - 3' \\
& Reverse, 5'-GAC TTC CCC GCA CCG ATT CC-3'
\end{tabular}

Daltonik, Germany). We overlaid dried spots with MALDI matrix $(10 \mathrm{mg} / \mathrm{mL} \alpha$-cyano-4-hydroxy-cinnamic acid $[\alpha-\mathrm{HCCA}]$ in $50 \%$ acetonitrile:2.5\% trifluoroacetic acid) (Bruker Daltonik, Germany). After drying of the matrix, we conducted MALDI-TOF MS analysis with a Microflex LT/SH benchtop mass spectrometer (Bruker Daltonik, Germany) equipped with a $60-\mathrm{Hz}$ nitrogen laser. We had optimized parameter settings (ion source 1 [IS1], $20 \mathrm{kV}$; IS2, $18.2 \mathrm{kV}$; lens, $6.85 \mathrm{kV}$; detector gain, $2854 \mathrm{~V}$; gating, none) for the mass range between 2000 and 20,000 Da. We achieved spectra in the positive linear mode with the maximum laser frequency. An external standard (bacterial test standard [BTS]) (Bruker Daltonik, Germany) was applied for instrument calibration. Data evaluation was performed by visually comparing spectra to search for peak shifts using flexAnalysis 3.4 (Bruker Daltonik, Germany).

\section{PCR amplification and DNA sequencing}

DNA was extracted from cultured colonies using the DNeasy UltraClean Microbial Kit (QIAGEN, Germany), and PCR was conducted using Ex Taq DNA polymerase, hot-start version (Takara, Japan) according to the manufacturer's instructions. The gene-specific primer pairs used for PCR analysis are listed in Table 4; these primers were used in previous studies [23, 24]. The sequencing PCR products were purified with the BigDye XTerminator purification kit (Life Technologies, USA) and samples were loaded on the ABI Prism 3130 Genetic Analyzer (Thermo Fisher Scientific, USA). The DNA sequencing results were analyzed using a BLAST search to identify sequence similarity between samples and the three species of $M$. abscessus complex.

\section{Antimicrobial susceptibility testing}

Susceptibility testing was performed according to Clinical and Laboratory Standard Institute (CLSI) guideline M24-A2 [25]. MIC determinations and synergy testing were performed by the checkerboard method using frozen broth microdilution plates (Eiken Chemical Co., Ltd., Japan). The ranges of antibiotic concentrations tested were as follows: amikacin (AMK) 0.25 to $64 \mu \mathrm{g} / \mathrm{mL}$, clarithromycin (CLR) 0.06 to $64 \mu \mathrm{g} / \mathrm{mL}$, imipenem (IPM) 4 to $32 \mu \mathrm{g} / \mathrm{mL}$, and moxifloxacin (MXF) 1 to $32 \mu \mathrm{g} / \mathrm{mL}$. MICs of each antimicrobial agent were determined by broth microdilution methods as recommended by the CLSI. The panels were prepared with a 96-channel dispenser and stored at $-80^{\circ} \mathrm{C}$ until use. CLR were dispensed alone in the first row, and IPM, AMK, or MXF were dispensed in the first column. Each well was inoculated with a concentration of $1 \times 10^{5}$ colony-forming units (CFU)/ $\mathrm{mL}$. The MICs were determined after 3, 7, 14 days of incubation at $35^{\circ} \mathrm{C}$. The $\mathrm{MIC}$ breakpoints, indicating susceptible, intermediate, and resistant strains, were interpreted according to the CLSI criteria for amikacin, cefoxitin, ciprofloxacin, clarithromycin, doxycycline, imipenem, linezolid, moxifloxacin, trimethoprim/ sulfamethoxazole, and tobramycin (Table 5) [25]. The effect of each agent combined with clarithromycin was evaluated using FIC index analysis [13].

\section{Statistical analysis}

Categorical variables were compared using the chisquare test or Fisher's exact test. The evaluation of changes in MIC was performed using the Wilcoxon

Table 5 Antimicrobial agents and MIC breakpoints for rapidly growing mycobacteria

\begin{tabular}{|c|c|c|c|}
\hline \multirow[b]{2}{*}{ Antimicrobial agents } & \multicolumn{3}{|c|}{ MIC $(\mu \mathrm{g} / \mathrm{mL})$ for category } \\
\hline & Susceptible & Intermediate & Resistant \\
\hline Amikacin & $\leq 16$ & 32 & $\geq 64$ \\
\hline Cefoxitin & $\leq 16$ & $32-64$ & $\geq 128$ \\
\hline Ciprofloxacin & $\leq 1$ & 2 & $\geq 4$ \\
\hline Clarithromycin & $\leq 2$ & 4 & $\geq 8$ \\
\hline Doxycycline & $\leq 1$ & $2-4$ & $\geq 8$ \\
\hline Imipenem & $\leq 4$ & $8-16$ & $\geq 32$ \\
\hline Linezolid & $\leq 8$ & 16 & $\geq 32$ \\
\hline Moxifloxacin & $\leq 1$ & 2 & $\geq 4$ \\
\hline Trimethoprim- sulfamethoxazole & $\leq 2 / 38$ & - & $\geq 4 / 76$ \\
\hline Tobramycin & $\leq 2$ & 4 & $\geq 8$ \\
\hline
\end{tabular}


signed-rank test. Differences were considered significant at $p<0.05$. When the chi-square test results were statistically significant, adjusted residuals were calculated to determine which particular associations were significant. Adjusted residuals were significant at $p<0.05$ level if they were less than -1.96 or more than 1.96 , and were significant at $p<0.01$ level if they were less than -2.58 or more than 2.58. All statistical analyses were performed using the SPSS software program (version 20, IBM Japan, Japan).

\section{Supplementary information}

Supplementary information accompanies this paper at https://doi.org/10. 1186/s12866-020-02000-5.

\section{Additional file 1: Supplementary figure 1. MIC distributions for} amikacin, imipenem, and moxifloxacin combined with clarithromycin, categorized into three subspecies of M. abscessus complex on day 3. Green color indicates susceptibility, yellow color indicates intermediate, and red color indicates resistance to $M$. abscessus. Abbreviations: CLR, clarithromycin; AMK, amikacin; IPM, imipenem; MXF, moxifloxacin; NA, not assessed. Supplementary figure 2. MIC distributions for amikacin, imipenem, and moxifloxacin combined with clarithromycin, categorized into three subspecies of M. abscessus complex on day 14. Green color indicates susceptibility, yellow color indicates intermediate, and red color indicates resistance to M. abscessus. Abbreviations: CLR, clarithromycin; AMK, amikacin; IPM, imipenem; MXF, moxifloxacin. Supplementary Table 1. The changes of median MIC of clarithromycin and imipenem between monotherapy and combination therapy.

\section{Abbreviations}

NTM: Nontuberculous mycobacteria; M. abscessus: Mycobacteroides abscessus; CF: Cystic fibrosis; ATS/IDSA: American Thoracic Society/Infectious Diseases Society of America; FIC: Fractional inhibitory concentration; MGIT: Mycobacteria growth indicator tube; DDH: DNA-DNA hybridization; MALDI-TOF MS: Matrix-assisted laser-desorption/ionization time-of-flight mass spectrometry; BTS: Bacterial test standard; CLSI: CLINICAL and Laboratory Standard Institute; AMK: Amikacin; CLR: Clarithromycin; IPM: Imipenem; MXF: Moxifloxacin; HIV: Human immunodeficiency virus; CFU: Colony-forming units

\section{Acknowledgements}

The authors would like to thank the staff of Juntendo University Hospital for their contribution in collecting data.

\section{Authors' contributions}

Conceived and designed the experiments: ST1, HI, ST2, and AN. Performed the experiments: ST1. Analyzed the data: ST1, HI, ST2, and AN. Collected the data and/or samples: ST1, YF, JW, KK, KS, IS, YO, MI, MC, and SM. Contributed reagents/materials/analysis tools: ST1, TO, MC, and SM. Reviewed the initial and final drafts of the manuscript: $\mathrm{AO}$ and KT. Wrote the paper: ST1, HI, ST2, and AN. All authors read and approved the final manuscript.

\section{Funding}

This work was supported by the Grant for Cross-disciplinary Collaboration, Juntendo University (grant no. 2019-46 to T. Okabe).

\section{Availability of data and materials}

The datasets during the current study available from the corresponding author on reasonable request.

\section{Ethics approval and consent to participate}

The study was approved by the Independent Ethics Committee at Juntendo University Hospital (approval no. 18-010 and 19-038) and adhered to the tenets of the Declaration of Helsinki.
Consent for publication

Not applicable.

\section{Competing interests}

The authors declare that they have no competing interests.

\section{Author details}

'Department of Clinical Laboratory, Juntendo University, Faculty of Medicine \& Graduate School of Medicine, Tokyo, Japan. ${ }^{2}$ Department of Respiratory Medicine, Juntendo University, Faculty of Medicine \& Graduate School of Medicine, 2-1-1 Hongo, Bunkyo-Ku, Tokyo 113-8421, Japan. ${ }^{3}$ Koto Hospital, Tokyo, Japan. ${ }^{4}$ Research Institute for Diseases of Old Ages, Juntendo University, Faculty of Medicine \& Graduate School of Medicine, Tokyo, Japan. ${ }^{5}$ Juntendo Tokyo Koto Geriatric Medical Center, Tokyo, Japan. ${ }^{6}$ Leading Center for the Development and Research of Cancer Medicine, Juntendo University, Faculty of Medicine \& Graduate School of Medicine, Tokyo, Japan. ${ }^{7}$ Department of Transfusion Medicine and Stem Cell Regulation, Juntendo University, Faculty of Medicine \& Graduate School of Medicine, Tokyo, Japan.

Received: 31 May 2020 Accepted: 9 October 2020

Published online: 19 October 2020

\section{References}

1. Bodle EE, Cunningham JA, Della-Latta P, Schluger NW, Saiman L. Epidemiology of nontuberculous mycobacteria in patients without HIV infection, New York city. Emerg Infect Dis. 2008;14(3):390-6.

2. Prevots DR, Shaw PA, Strickland D, et al. Nontuberculous mycobacterial lung disease prevalence at four integrated health care delivery systems. Am J Resp Crit Care. 2010;182(7):970-6.

3. Namkoong $\mathrm{H}$, Kurashima A, Morimoto $\mathrm{K}$, et al. Epidemiology of pulmonary Nontuberculous mycobacterial disease, Japan. Emerg Infect Dis. 2016;22(6): $1116-7$

4. Roux AL, Catherinot E, Ripoll F, et al. Multicenter study of prevalence of nontuberculous mycobacteria in patients with cystic fibrosis in France. J Clin Microbiol. 2009;47(12):4124-8.

5. Olivier KN, Weber DJ, Wallace RJ Jr, et al. Nontuberculous mycobacteria. I: multicenter prevalence study in cystic fibrosis. Am J Respir Crit Care Med. 2003;167(6):828-34.

6. Swenson JM, Wallace RJ Jr, Silcox VA, Thornsberry C. Antimicrobial susceptibility of five subgroups of Mycobacterium fortuitum and Mycobacterium chelonae. Antimicrob Agents Chemother. 1985;28(6):807-11.

7. Brown BA, Wallace RJ Jr, Onyi GO, De Rosas V, Wallace RJ 3rd. Activities of four macrolides, including clarithromycin, against Mycobacterium fortuitum, Mycobacterium chelonae, and M. chelonae-like organisms. Antimicrob Agents Chemother. 1992;36(1):180-4.

8. Griffith DE, Aksamit T, Brown-Elliott BA, et al. An official ATS/IDSA statement: diagnosis, treatment, and prevention of nontuberculous mycobacterial diseases. Am J Respir Crit Care Med. 2007:175(4):367-416.

9. Novosad SA, Beekmann SE, Polgreen PM, Mackey K, Winthrop KL. Team MaS. Treatment of Mycobacterium abscessus infection. Emerg Infect Dis. 2016;22(3):511-4.

10. Bastian S, Veziris N, Roux AL, et al. Assessment of clarithromycin susceptibility in strains belonging to the Mycobacterium abscessus group by erm (41) and rrl sequencing. Antimicrob Agents Ch. 2011;55(2):775-81.

11. Kim HY, Kim BJ, Kook Y, et al. Mycobacterium massiliense is differentiated from Mycobacterium abscessus and Mycobacterium bolletii by erythromycin ribosome methyltransferase gene (erm) and clarithromycin susceptibility patterns. Microbiol Immunol. 2010;54(6):347-53.

12. Adjemian J, Prevots DR, Gallagher J, Heap K, Gupta R, Griffith D. Lack of adherence to evidence-based treatment guidelines for nontuberculous mycobacterial lung disease. Ann Am Thorac Soc. 2014;11(1):9-16.

13. Lorian V. Antibiotics in laboratory medicine. 4th ed. Baltimore: Williams \& Wilkins; 1996.

14. Ferro BE, Srivastava S, Deshpande D, et al. Failure of the Amikacin, Cefoxitin, and clarithromycin combination regimen for treating pulmonary Mycobacterium abscessus infection. Antimicrob Agents Chemother. 2016; 60(10):6374-6

15. Lerat I, Cambau E, Roth Dit Bettoni R, et al. In vivo evaluation of antibiotic activity against Mycobacterium abscessus. J Infect Dis. 2014;209(6):905-12.

16. Pasipanodya JG, Ogbonna D, Ferro BE, et al. Systematic Review and Metaanalyses of the Effect of Chemotherapy on Pulmonary Mycobacterium 
abscessus Outcomes and Disease Recurrence. Antimicrob Agents Chemother. 2017;61(11):e01206-17. https://doi.org/10.1128/AAC.01206-17.

17. Le Run $E$, Arthur M, Mainardi JL. In Vitro and Intracellular Activity of Imipenem Combined with Rifabutin and Avibactam against Mycobacterium abscessus. Antimicrob Agents Chemother. 2018;62(8):e00623-18. https://doi. org/10.1128/AAC.00623-18.

18. Lefebvre AL, Dubee V, Cortes M, Dorchene D, Arthur M, Mainardi JL. Bactericidal and intracellular activity of beta-lactams against Mycobacterium abscessus. J Antimicrob Chemother. 2016;71(6):1556-63.

19. Miyasaka T, Kunishima H, Komatsu M, et al. In vitro efficacy of imipenem in combination with six antimicrobial agents against Mycobacterium abscessus. Int J Antimicrob Agents. 2007;30(3):255-8.

20. Kim BJ, Yi SY, Shim TS, et al. Discovery of a Novel hsp65 Genotype within Mycobacterium massiliense Associated with the Rough Colony Morphology. Plos One. 2012;7(6):e38420. https://doi.org/10.1371/journal.pone.0038420.

21. Yoshida S, Tsuyuguchi K, Suzuki K, et al. Further isolation of Mycobacterium abscessus subsp abscessus and subsp bolletii in different regions of Japan and susceptibility of these isolates to antimicrobial agents. Int J Antimicrob Ag. 2013;42(3):226-31.

22. Sparbier K, Lange $C$, Jung J, et al. MALDI Biotyper-based rapid resistance detection by stable-isotope labeling. J Clin Microbiol. 2013;51(11):3741-8.

23. Nakanaga K, Sekizuka T, Fukano H, et al. Discrimination of Mycobacterium abscessus subsp. massiliense from Mycobacterium abscessus subsp. abscessus in clinical isolates by multiplex PCR. J Clin Microbiol. 2014;52(1): 251-9.

24. Brown-Elliott BA, Vasireddy S, Vasireddy $\mathrm{R}$, et al. Utility of sequencing the erm (41) gene in isolates of Mycobacterium abscessus subsp. abscessus with low and intermediate clarithromycin MICs. J Clin Microbiol. 2015;53(4): $1211-5$.

25. Woods GL, Brown-Elliott BA, Conville PS, et al. In: nd, ed. Susceptibility Testing of Mycobacteria, Nocardiae, and Other Aerobic Actinomycetes. Wayne: Clinical and Laboratory Standards Institute; 2011.

\section{Publisher's Note}

Springer Nature remains neutral with regard to jurisdictional claims in published maps and institutional affiliations.

Ready to submit your research? Choose BMC and benefit from:

- fast, convenient online submission

- thorough peer review by experienced researchers in your field

- rapid publication on acceptance

- support for research data, including large and complex data types

- gold Open Access which fosters wider collaboration and increased citations

- maximum visibility for your research: over $100 \mathrm{M}$ website views per year

At $\mathrm{BMC}$, research is always in progress.

Learn more biomedcentral.com/submissions 